\title{
Multiphysics dynamic simulation of the E-ELT M4 adaptive mirror during earthquake
}

\author{
Manetti Mauro $^{\text {a }}$, Tintori Matteo ${ }^{b}$, Biasi Roberto ${ }^{a}$, Mantegazza Marco ${ }^{b}$ \\ ${ }^{a}$ Microgate Srl, via Stradivari, 4 - 39100 Bolzano-Bozen (BZ) - Italy \\ ${ }^{\mathrm{b}}$ A.D.S. International Srl, via Roma, 87 - 23868 Valmadrera (LC) - Italy
}

\begin{abstract}
Most of the future extremely large telescopes will be built in seismic areas, heavily affected by earthquakes. For example, the E-ELT is located at Cerro Armazones, in Chile, where earthquakes, also with high magnitudes, are a well-known reality. This is the reason why the telescope, together with all its instruments and facilities, is required to survive seismic events. The focus of this paper is on the dynamic simulation of the E-ELT M4 adaptive mirror during earthquake.

The M4 adaptive mirror is actuated through a contact-less technology based on voice coil motors and the mirror is held to the telescope only through a membrane and bias magnets. The in-plane displacements and the rotation around the axis normal to the reflective surface are well restrained by the membrane, which is designed to survive earthquakes. On the contrary the shell piston displacement and tip-tilt rotations, when the system is in off state, are constrained only by bias magnets which pull the mirror against the reference body. However, during a strong seismic activity, the bias magnet forces could not be sufficient to avoid the shell detachment, so the voice coil motors must be activated to provide an additional retention force. The present work shows the development of a multiphysics simulator, which is able to reproduce the shell dynamic behavior along its normal direction during earthquake, so that appropriate levels of forces provided by the actuators and appropriate system safety strategies can be designed as function of the earthquake intensity.
\end{abstract}

Keywords: E-ELT, adaptive optics, deformable mirror, earthquake simulation

\section{EARTHQUAKE ACCELERATION TIME HISTORIES}

Three statistically independent set of acceleration time histories at the telescope ground have been computed by ESO, following the European code guidelines [1]. From ground accelerations, ESO has derived the accelerations at the interface between the telescope and the M4 assembly, considering possible dynamic amplifications because of the telescope structure.

The further dynamic amplification introduced by the E-ELT M4 assembly (hexapod, reference body, connection system) has been evaluated through the FE dynamic analysis of an appropriate simplified model. Through the numerical integration of the dynamic equations of the FE model, we obtained the piston and tip/tilt rigid displacements of the reference body at the connection of the M4 mirror retention membranes. These rigid displacements represent the Dirichlet boundary conditions for the mirror dynamic analysis during earthquake. The elastic deformations of the reference body induced by earthquake have not been taken into account because considered negligible with respect to the elastic deformations of the mirror. Figure 1 (left) shows the piston displacement and tip/tilt rotations of the reference body, induced by the first set of accelerations provided by ESO, while Figure 1 (right) shows the corresponding linear and angular accelerations. 

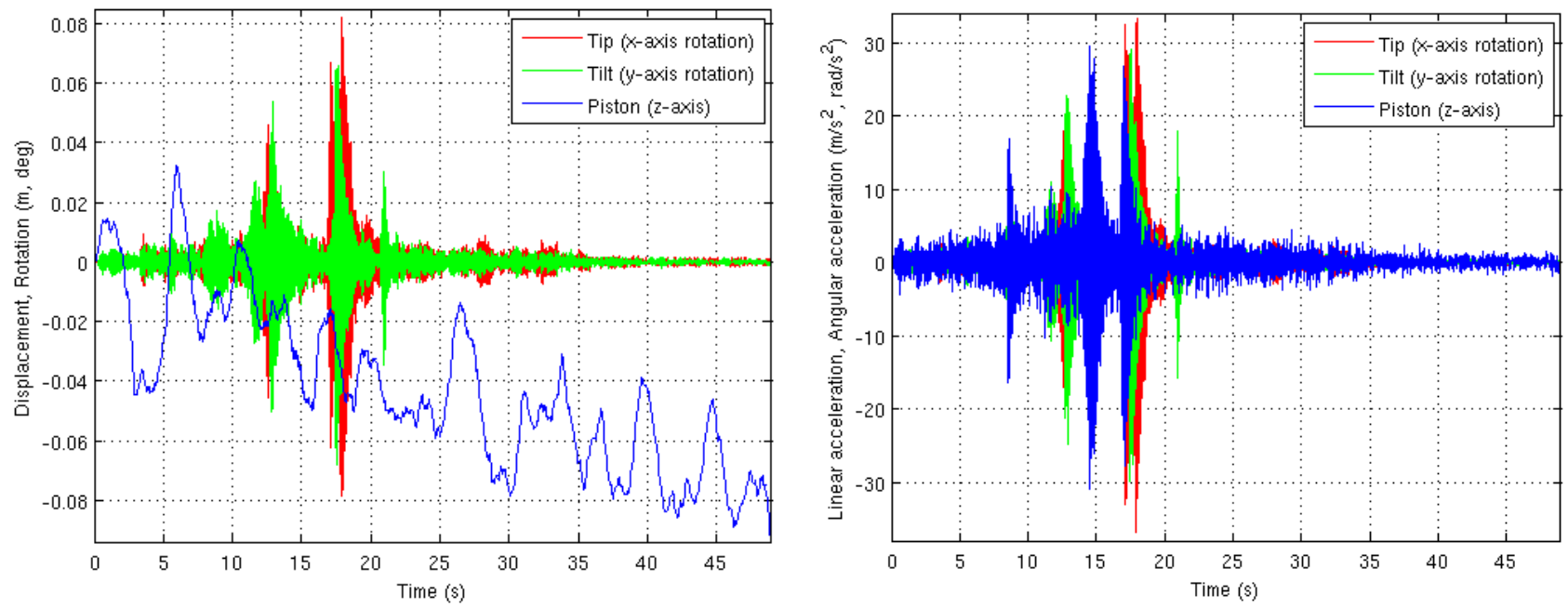

Figure 1: E-ELT M4 reference body piston displacement and tip/tilt rotations due to the first acceleration set provided by ESO (left), reference body linear piston acceleration and tip/tilt angular accelerations due to the first acceleration set provided by ESO (right).

\section{M4 MIRROR SIMULATION MODEL}

The M4 mirror numerical model takes into account [2][3]: the fluid dynamics of the air trapped between the reference body and the mirror, the structural dynamics of the mirror (normal modes representation), the servo-components such as voice coil motor actuators and capacitive position sensors.

The multiphysics-coupled simulator described in [2] and [3] represents the starting code to run the earthquake analysis here presented. However, the simulation of the mirror response during earthquake requires the introduction of some new features:

1. the simulator must handle the mirror rigid body motions, piston and tip/tilt;

2. the earthquake induced accelerations must be applied to the system by imposing the displacements at the interface between membranes and reference body, so through the application of boundary conditions.

As already underlined, the reference body is here approximated as a rigid body with three degrees of freedom, piston and tip/tilt, because the mirror elastic deformation is considered highly dominant. The displacements of the membranes are simply imposed through the introduction of "dummy" ideal sensor/actuator units, controlled in force through a closed loop characterized by very high proportional gains. In this way the required displacements, in the range of several centimeters, can be set with an accuracy in the order of few nanometers and the simulation code modifications required to handle an earthquake condition are quite limited. Possible collisions between the reference body and the mirror during the shacking introduced by earthquake are not modeled inside the code, i.e. no contact modeling. Therefore, to avoid numerical problems of the fluid dynamic model, which becomes singular with null or negative air gaps, a nonlinear cubic spring has been added under each "real" actuation point, which furnishes a fictitious elastic force below a user defined air reference gap, set at $30 \mu \mathrm{m}$ for the analysis here presented. Higher this reference gap, higher is conservative the solution because of lower air damping. Figure 2 shows the fluid dynamic mesh of the model on the left, and the layout of the actuation points on the right. The blue actuation points are the "real" system actuators required to control the mirror shape, while the red points represents the "dummy" actuators introduced to impose the membrane displacements, i.e. the appropriate boundary conditions of the model. 

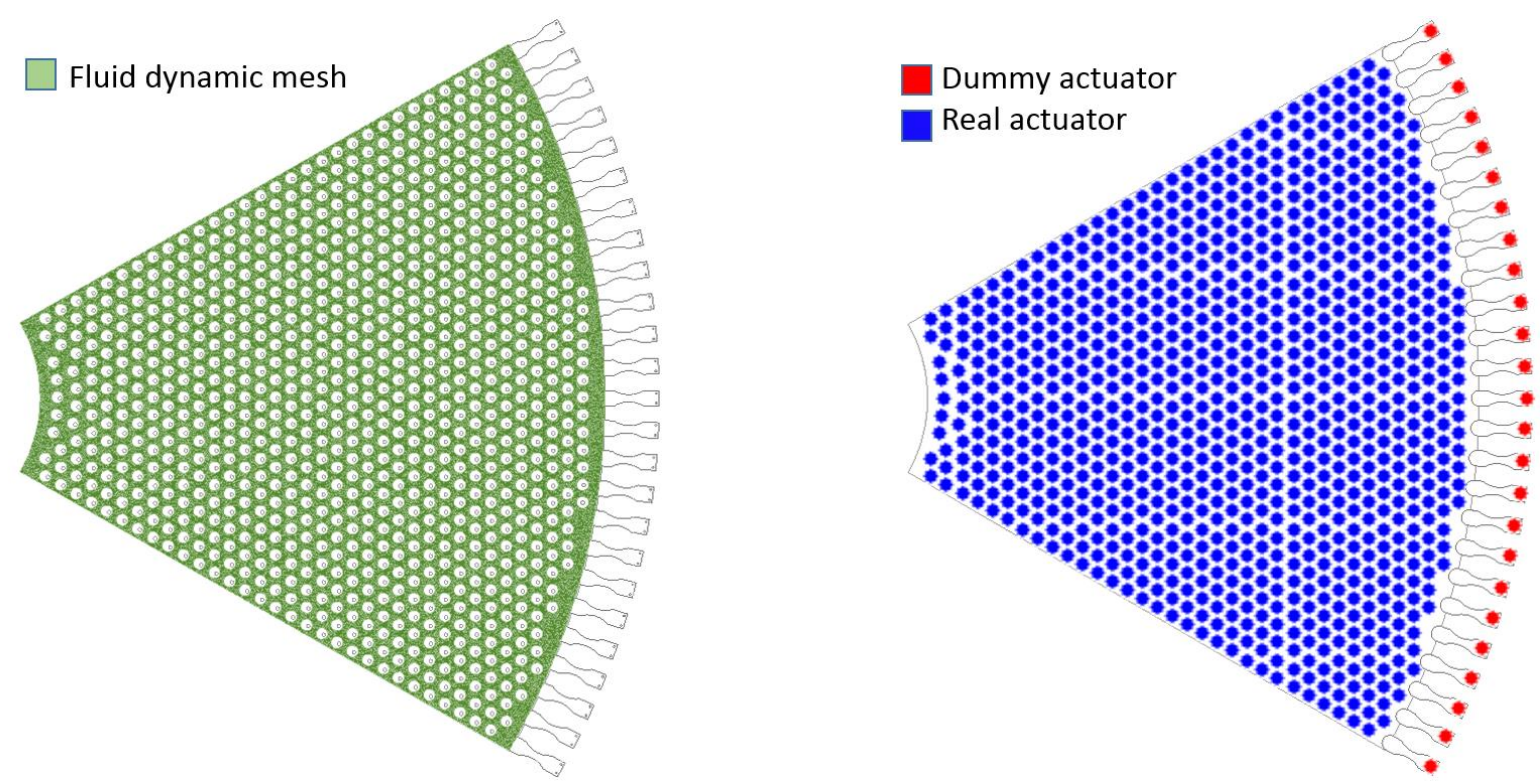

Figure 2: Fluid dynamic mesh of the E-ELT M4 mirror multiphysics model (left), layout of the "dummy" and "real" actuation points of the model (right).

\section{ANALYSIS RESULTS}

The simulator allows verifying the system behavior during a seismic event. The most critical condition happens when the mirror is not actively controlled, but in an off-state. In fact, when not active, the mirror is only retained along the direction normal to the reflective surface by passive forces provided by bias magnets, which pull it against the reference body with a force which is about two times the weight of the mirror itself. In case of earthquake, an additional open loop retention force can be added through the activation of the voice coil motors. The voice coil motor activation requires about 50ms. The main goal of the present analysis is to identify the values of such additional force as function of the earthquake intensity, i.e. acceleration, which is measured through accelerometers. Some aspects are worth of noticing:

1. the passive force distribution is set linear along the radial shell direction, but constant within the actuators belonging to the same electro-mechanical unit, that is called brick; a brick is the basic electro-mechanical unit of the E-ELT M4 system, which groups together a variable number of actuators, typically 28 or 36 actuators (see [4] for more details); this spatial force distribution allows to optimize the shell retention because the accelerations experienced by the system are higher on the external border;

2. the open-loop additional retention force when the system is in off-state is applied with a delay of about 50ms with respect to the critical acceleration detection;

3. the average air gap between the reference body and the mirror when the system is in off-state can be estimated in the order of $20-25 \mu \mathrm{m}$ (conservative assumption); in this condition the air damping is not at all negligible and highly affects the system dynamics, mainly helping the shell retention against the reference body; however, this damping force is highly nonlinear and rapidly decreases (cubic behavior) with higher gaps.

The acceleration induced by earthquake is measured on the system through accelerometers. Every brick of the system mounts one accelerometer. When an accelerometer measures an acceleration that exceeds an established threshold, a predetermined set of passive retention forces are applied to the system. The aim of present simulations is to design the acceleration thresholds value and the relative passive retention force set through a trial and error procedure. The goal is to avoid the mirror detachment, so the shell should be kept as close as possible to the reference body.

The most stressed area of the mirror in terms of acceleration is the external crown, because the piston linear acceleration sums up to tip-tilt angular accelerations. Therefore, to design the time instants where passive retention forces must be 
switched on during simulation, it is exploited the mean acceleration of the system over a prescribed external crown of actuation points, as shown in Figure 3 (left). This is a conservative approach, because the "punctual" measurement of the accelerometers mounted on bricks usually experience/detect the acceleration thresholds in advance with respect to the mean acceleration computed in this way. At the end of the trial and error procedure, two acceleration thresholds have been designed, at $0.3 \mathrm{~g}$ and $1.0 \mathrm{~g}$ respectively. Figure 3 (right) shows the mean acceleration induced on the system by the first time history provided by ESO; the time instants where the mean acceleration exceeds $0.3 \mathrm{~g}$ and $1.0 \mathrm{~g}$ are highlighted. At each threshold corresponds the application to the system of a prescribed set of forces. These forces are shown in Figure 4 , left and right for the thresholds of $0.3 \mathrm{~g}$ and $1.0 \mathrm{~g}$ respectively. The maximum and minimum values of the passive retention forces are reported in Table 1.
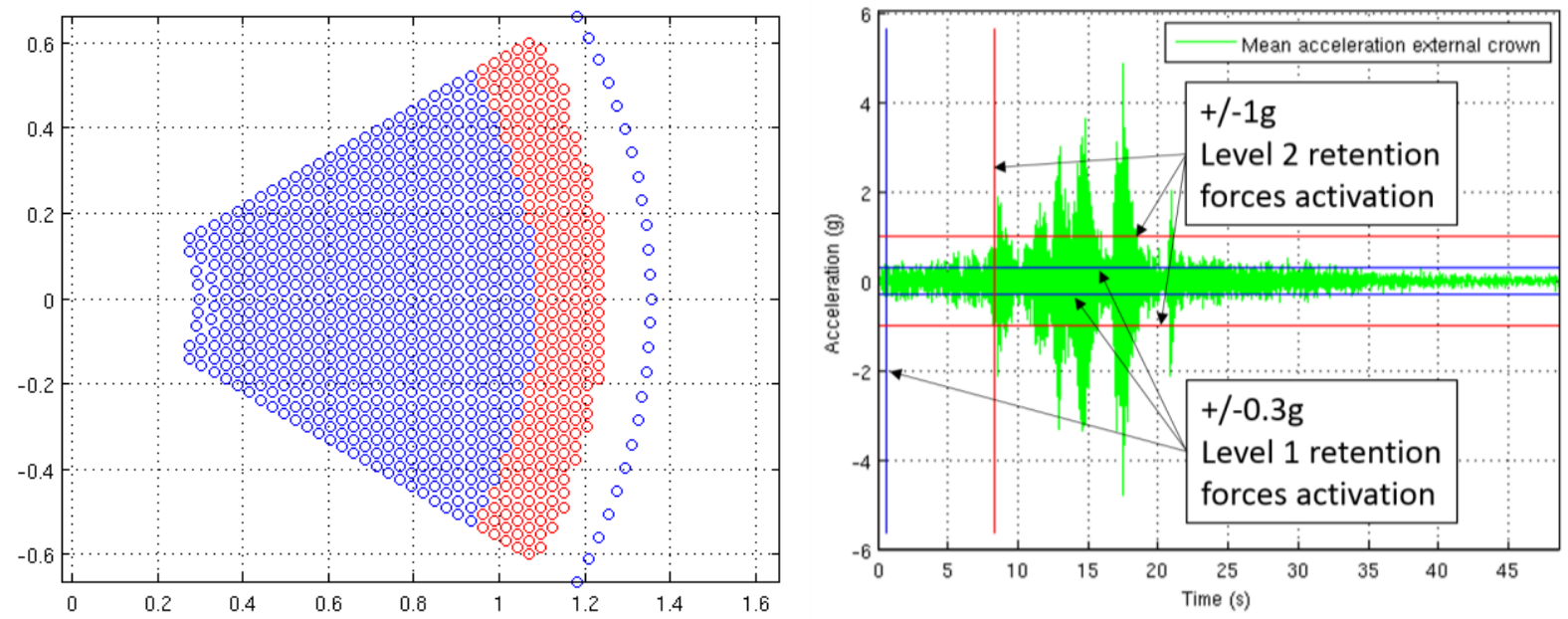

Figure 3: External crown of actuation points where the mean acceleration is computed (left); mean acceleration time history with the time instants where the acceleration thresholds are exceeded and the passive retention forces activated.
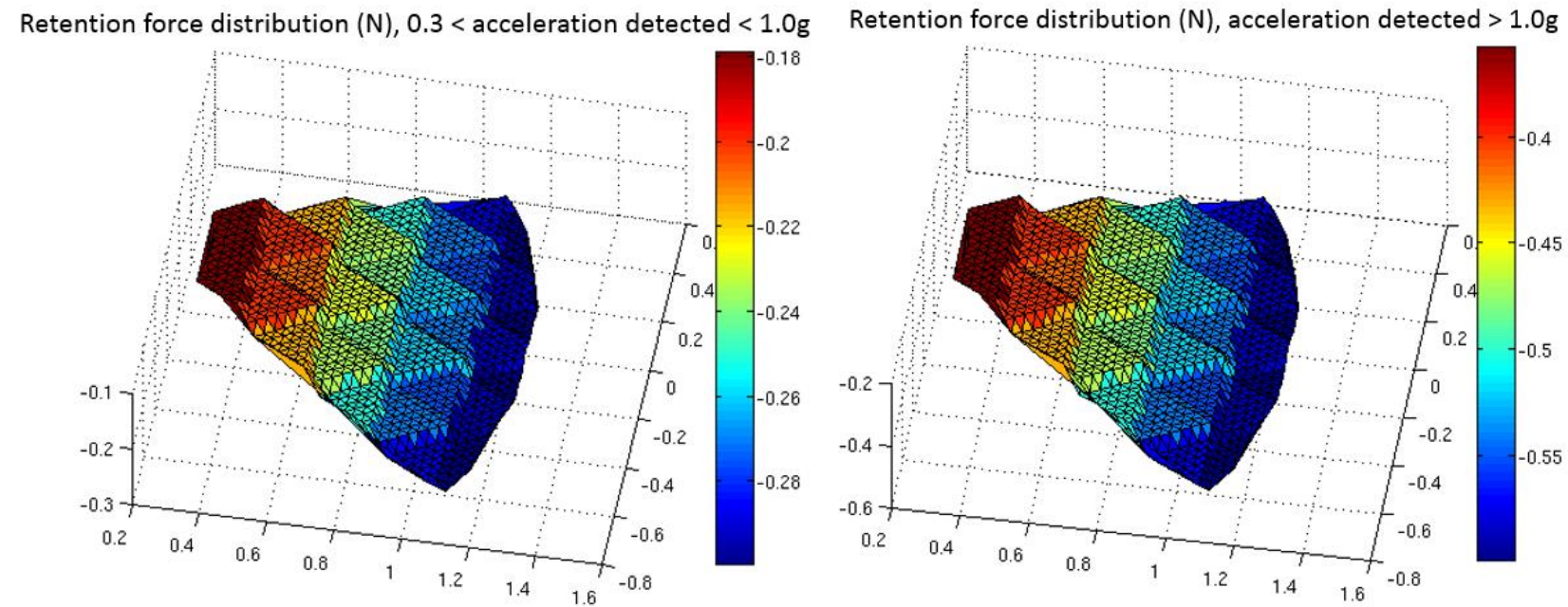

Figure 4: Passive retention force set applied for acceleration higher than $0.3 \mathrm{~g}$ and lower $1.0 \mathrm{~g}$ (left); passive retention force set applied for acceleration higher than $1.0 \mathrm{~g}$ (right). 
Table 1: Final acceleration thresholds and passive retention forces min and max values.

\begin{tabular}{|c|c|c|}
\hline Acceleration level $(\mathbf{g})$ & Min force $(\mathbf{N})$ & Max force $(\mathbf{N})$ \\
\hline$<0.3$ & 0.18 & 0.36 \\
\hline$<1.0$ & 0.30 & 0.60 \\
\hline
\end{tabular}

Figure 5 shows the time history of the gap between the mirror and the reference body during the earthquake at each actuation point. This plot refers to the first set of earthquake acceleration provided by ESO. Considering the presence of the artificial cubic spring working below $30 \mu \mathrm{m}$, the results confirm the soundness of the passive retention forces and acceleration thresholds designed. Similar results have been obtained with the other two accelerations set provided by ESO.

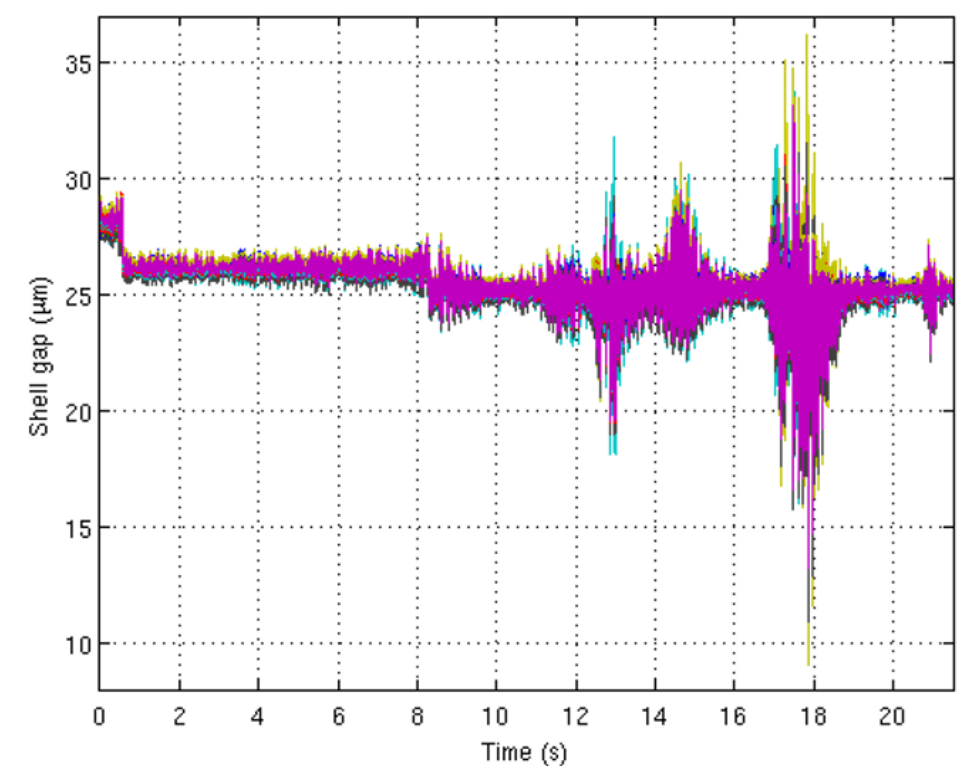

Figure 5: E-ELT M4 mirror gap at actuation points between mirror and reference body during earthquake.

\section{FINAL REMARKS}

The simulations performed allowed to design the values of the open-loop retention force as function of the earthquake intensity, such that the shell detachment can be avoided. Two different levels of forces have been implemented as function of two increasingly earthquake accelerations detected:

- $\quad 0.3 \mathrm{~g}, \min$ retention force $0.18 \mathrm{~N}$, max retention force $0.3 \mathrm{~N}$;

- $\quad 1.0 \mathrm{~g}$, min retention force $0.36 \mathrm{~N}, \max$ retention force $0.6 \mathrm{~N}$.

The very same high fidelity simulation could be exploited in future to further optimize the design of the earthquake shell safety system, but also to evaluate the mirror behavior in presence of vibrations of the support structure. 


\section{REFERENCES}

[1] "Eurocode 8: Design of structures for earthquake resistance", EN 1998-1:2004

[2] Manetti, M., Morandini, M., Mantegazza, P., "Servo-Fluid-Elastic Modeling of Contactless Levitated Adaptive Secondary", Computational Mechanics 50 (1), 85-98 (2012).

[3] Manetti, M., Morandini, M., Mantegazza, P., Biasi, R., Gallieni, D., Riccardi, A., "Modeling and control of massively actuated, magnetically levitated, adaptive mirrors" In IEEE international conference on control applications (CCA) (pp. 860-866) (2010).

[4] Gallieni, D., Tintori, M., Mantegazza, M., Anaclerio, E., Crimella, L., Acerboni, M., Biasi, R., Angerer, G., Andrigettoni, M., Merler, A., Veronese, D., Carel, J.-L., Marque, G., Molinari, E., Tresoldi, D., Toso, G., Spanó, P., Riva, M., Mazzoleni, R., Riccardi, A., Mantegazza, P., Manetti, M., Morandini, M., Vernet, E., Hubin, N., Jochum, L., Madec, P., Dimmler, M., Koch, F., "Voice-coil technology for the E-ELT M4 Adaptive Unit", Presented at the Adaptative Optics for Extremely Large Telescopes, (2010). 